\title{
Chest radiograph features of multisystem inflammatory syndrome in children (MIS-C) compared to pediatric COVID-19
}

\author{
Bradley S. Rostad ${ }^{1,2}$ (1) Jay H. Shah ${ }^{1,2} \cdot$ Christina A. Rostad ${ }^{2,3,4}$. Preeti Jaggi $i^{2,3,4} \cdot$ Edward J. Richer $^{1,2}$ • \\ Leann E. Linam ${ }^{1,2}$. Adina L. Alazraki ${ }^{1,2} \cdot$ Erica L. Riedesel ${ }^{1,2} \cdot$ Sarah S. Milla ${ }^{1,2}$
}

Received: 14 July 2020 / Revised: 28 September 2020 / Accepted: 22 November 2020 / Published online: 6 January 2021

(C) Springer-Verlag GmbH Germany, part of Springer Nature 2021

\begin{abstract}
Background Although the radiographic features of coronavirus disease 2019 (COVID-19) in children have been described, the distinguishing features of multisystem inflammatory syndrome in children (MIS-C) associated with COVID-19 are not well characterized.

Objective We compared the chest radiographic findings of MIS-C with those of COVID-19 and described other distinguishing imaging features of MIS-C.

Materials and methods We performed a retrospective case series review of children ages 0 to 18 years who were hospitalized at Children's Healthcare of Atlanta from March to May 2020 and who either met the Centers for Disease Control and Prevention (CDC) case definition for MIS-C $(n=11)$ or who had symptomatic, laboratory-confirmed COVID-19 $(n=16)$. Two radiologists reviewed the most severe chest radiographs for each patient. The type and distribution of pulmonary opacities and presence or absence of pleural effusions were recorded. The chest radiographs were categorized based on potential COVID-19 imaging findings as typical, indeterminate, atypical or negative. An imaging severity score was also assigned using a simplified version of the Radiographic Assessment of Lung Edema Score. Findings were statistically compared between patients with MIS-C and those with COVID-19. Additional imaging findings of MIS-C were also described.

Results Radiographic features of MIS-C included pleural effusions ( $82 \%$ [9/11]), pulmonary consolidations $(73 \%$ [8/11]) and ground glass opacities $(91 \%$ [10/11]). All of the lung opacities $(100 \%$ [10/10]) were bilateral, and the majority of the pleural effusions (67\% [6/9]) were bilateral. Compared to children with COVID-19, children with MIS-C were significantly more likely to develop pleural effusions on chest radiograph $(82 \%[9 / 11]$ vs. $0 \%[0 / 0], P$-value $<0.01)$ and a lower zone predominance of pulmonary opacifications $(100 \%$ [10/10] vs. $38 \%$ [5/13], $P$-value $<0.01)$. Children with MIS-C who also had abdominal imaging had intra-abdominal inflammatory changes.

Conclusion Key chest radiographic features of MIS-C versus those of COVID-19 were pleural effusions and lower zone pulmonary opacifications as well as intra-abdominal inflammation. Elucidating the distinguishing radiographic features of MIS-C may help refine the case definition and expedite diagnosis and treatment.
\end{abstract}

Keywords Abdomen · Chest · Children · Computed tomography · Coronavirus disease $2019 \cdot$ Multisystem inflammatory syndrome in children $\cdot$ Radiography $\cdot$ Ultrasound

Bradley S. Rostad

brostad@emory.edu

1 Department of Radiology and Imaging Sciences,

Emory University School of Medicine,

1405 Clifton Rd. NE, Atlanta, GA 30322, USA

2 Children's Healthcare of Atlanta,

Atlanta, GA, USA
3 Department of Pediatrics,

Division of Pediatric Infectious Diseases,

Emory University School of Medicine,

Atlanta, GA, USA

4 Center for Childhood Infections and Vaccines, Children's Healthcare of Atlanta and Emory University School of Medicine, Atlanta, GA, USA 


\section{Introduction}

Severe acute respiratory syndrome coronavirus 2 (SARS$\mathrm{CoV}-2)$ is the novel betacoronavirus responsible for coronavirus disease 2019 (COVID-19). This virus first emerged in Wuhan, China, in December 2019 and has since been declared a pandemic by the World Health Organization (WHO) [1]. Although severe disease disproportionately affects older adults and individuals with underlying comorbidities, a spectrum of disease severity has also been observed in a minority of children [2]. Recently, a multisystem inflammatory syndrome in children (MIS-C) temporally associated with COVID-19 has been described [3-6]. Children with this syndrome have had either epidemiologic exposure to COVID-19 or evidence of current or preceding SARS-CoV-2 infection by positive polymerase chain reaction (PCR) or serologic testing [7]. They have presented with signs of hyperinflammation and multi-organ dysfunction $[8,9]$, often developing myocarditis [10] and cardiorespiratory failure, and occasionally dying [5, 6]. Although the radiographic features of COVID-19 in children have been characterized [11], data describing the radiographic findings of MIS-C are limited [12].

In this retrospective case series, we reviewed chest radiographs of children hospitalized with either MIS-C or COVID19 and categorized their imaging findings according to a recently proposed classification system for describing chest imaging findings in COVID-19 $[13,14]$. We statistically compared chest imaging findings of children with MIS-C versus COVID-19 to identify distinguishing radiographic features of these two entities, which have overlapping clinical features. Differentiating MIS-C from COVID-19 and other diagnoses with similar clinical appearances early in the disease course could help guide patient management and improve outcomes.

\section{Materials and methods}

\section{Institutional review board approval}

This retrospective study was approved by the institutional review board of Children's Healthcare of Atlanta with a waiver of informed consent. This study was conducted in compliance with the Health Insurance Portability and Accountability Act (HIPAA).

\section{Study population}

Patients 0 to 18 years of age admitted to Children's Healthcare of Atlanta with a diagnosis of either COVID19 or MIS-C from March through May 2020 were included. Children were classified as having COVID-19 if they were symptomatic and tested positive for SARS-CoV-2 by nasopharyngeal PCR. Children were classified as having MIS-C if they met the Centers for Disease Control and Prevention (CDC) case definition [7] and tested positive for SARSCoV-2 by either nasopharyngeal PCR or by nucleocapsid protein antibodies in the serum (Abbott Laboratories, Abbott Park, IL). Demographic and clinical data were obtained from the electronic medical record (Epic Systems Corporation, Verona, WI).

\section{Imaging analysis}

The most severe radiograph for each patient, that is the radiograph with the most extensive pulmonary opacification, was selected by a fellowship-trained pediatric radiologist (B.S.R., with 4 years of post-fellowship experience and a Certificate of Added Qualification [CAQ] in pediatric radiology). These radiographs were then independently reviewed by two fellowship-trained pediatric radiologists (B.S.R. and J.H.S. [1 year of post-fellowship experience and CAQ eligible]), and discrepancies were resolved by joint review. The following features of the pulmonary opacities were recorded: type (consolidation or ground glass), laterality (left, right or bilateral), distribution (peripheral, central or neither) and zonal predominance (upper, lower or neither). The presence or absence of pleural effusions were also recorded. The chest radiographs were categorized as typical, indeterminate, atypical or negative, based on imaging findings that could be attributed to COVID-19 [13]. These categories were initially described for use with computed tomography (CT) in adults and were subsequently adapted for use in pediatric chest radiography by Foust et al. [14]. Typical indicates commonly reported chest radiograph findings of pediatric COVID-19. Indeterminate indicates nonspecific chest radiograph findings of pediatric COVID-19. Atypical indicates chest radiograph findings that are uncommon or not reported in pediatric COVID-19. Negative indicates no chest radiographic findings of pediatric pneumonia. Radiographs were assigned a severity score based on a simplified version of the Radiographic Assessment of Lung Edema Score, which has previously been used by Wong et al. [15] to describe chest radiograph findings in adults with COVID-19 [16]. A score of 0-4 was assigned to each lung depending on the extent of involvement by consolidation or ground glass opacity $(0=$ no involvement, $1=<25 \%$ involvement, $2=25-50 \%$ involvement, $3=50-75 \%$ involvement, $4=>75 \%$ involvement) [16]. The scores for each lung were added to produce the final severity score of $0-8$. The frequencies of radiographic findings between patients with MIS-C and those with COVID-19 were then statistically compared.

All other imaging studies performed for patients with MIS$\mathrm{C}$ were similarly independently reviewed by two fellowshiptrained pediatric radiologists (B.S.R. and S.S.M. [13 years of post-fellowship experience and a CAQ in pediatric radiology]). Imaging utilization and key findings were summarized. 


\section{Data analysis}

Statistical analysis was performed using GraphPad Prism version 8.0 (GraphPad Software, San Diego, CA). Frequencies were compared using chi-squared tests, and continuous variables were compared using Student's $t$-tests. $P$-values $\leq 0.05$ were considered statistically significant.

\section{Results}

\section{Baseline characteristics}

Baseline characteristics for the study population are shown in Table 1 . The majority of children hospitalized with MIS-C and COVID-19 were male (64\% [7/11] of patients with MIS-C and $63 \%$ [10/16] of patients with COVID-19). Children with MIS$\mathrm{C}$ were significantly younger than those with COVID-19 (mean age: 9.0 years [standard deviation (SD) 3.5] vs. 13.5 years [SD
5.2], $P$-value $=0.02)$. In terms of the clinical presentation, the majority of children with MIS-C and COVID-19 presented with fever (100\% [11/11] of patients with MIS-C and $88 \%$ [14/16] of patients with COVID-19). Children with MIS-C were significantly more likely than children with COVID-19 to present with vomiting $(100 \%[11 / 11]$ vs. $50 \%$ [8/16], $P$ value $<0.01)$ and abdominal pain $(73 \%[8 / 11]$ vs. $6 \%[1 / 16]$, $P$-value $<0.01)$, and less likely to present with respiratory symptoms $(27 \%$ [3/11] vs. $88 \%$ [14/16], $P$-value $<0.01)$. Whereas the majority of children with MIS-C were previously healthy with a normal body mass index (BMI) $(73 \%$ [8/11]), the majority of children with COVID-19 had underlying comorbidities $(81 \%$ [13/16]) including obesity $(63 \%$ [10/16]), asthma (19\% $[3 / 16])$, malignancy $(13 \%[2 / 16])$, neurological disease $(13 \%$ [2/16]) and cardiac disease (6\% [1/16]).

All children with MIS-C in our study developed fluidrefractory shock requiring volume resuscitation and vasoactive medications. All children with MIS-C had hypoalbuminemia, $82 \%(9 / 11)$ required intravenous albumin infusion and
Table 1 Demographics and presenting symptoms of children with multisystem inflammatory syndrome in children (MIS-C) and coronavirus disease 2019 (COVID-19)

\begin{tabular}{|c|c|c|c|}
\hline & MIS-C $(n=11)$ & COVID-19 $(n=16)$ & $P$-value \\
\hline Gender & & & $>0.99$ \\
\hline Male, $n(\%)$ & $7(64 \%)$ & $10(63 \%)$ & \\
\hline Female, $n(\%)$ & $4(36 \%)$ & $6(38 \%)$ & \\
\hline Age in years, mean (SD) & $9.0(3.5)$ & $13.5(5.2)$ & 0.02 \\
\hline \multicolumn{4}{|l|}{ Constitutional symptoms } \\
\hline Fever, $n(\%)$ & $11(100 \%)$ & $14(88 \%)$ & 0.50 \\
\hline Headache, $n(\%)$ & $4(36 \%)$ & $3(19 \%)$ & 0.39 \\
\hline Sore throat, $n(\%)$ & $0(0 \%)$ & $3(19 \%)$ & 0.25 \\
\hline Myalgia, $n(\%)$ & $2(18 \%)$ & $2(13 \%)$ & $>0.99$ \\
\hline Respiratory symptoms (any) & $3(27 \%)$ & $14(88 \%)$ & $<0.01$ \\
\hline Cough, $n(\%)$ & $3(27 \%)$ & $13(81 \%)$ & 0.02 \\
\hline Dyspnea, $n(\%)$ & $0(0 \%)$ & $6(38 \%)$ & 0.05 \\
\hline Chest pain, $n(\%)$ & $0(0 \%)$ & $3(19 \%)$ & 0.25 \\
\hline Abdominal symptoms (any) & $11(100 \%)$ & $10(63 \%)$ & 0.05 \\
\hline Vomiting, $n(\%)$ & $11(100 \%)$ & $8(50 \%)$ & $<0.01$ \\
\hline Diarrhea, $n(\%)$ & $7(64 \%)$ & $4(25 \%)$ & 0.06 \\
\hline Abdominal pain, $n(\%)$ & $8(73 \%)$ & $1(6 \%)$ & $<0.01$ \\
\hline Comorbidities (any) & $3(27 \%)$ & $13(81 \%)$ & 0.02 \\
\hline Obesity, $n(\%)$ & $1(9 \%)$ & $10(63 \%)$ & $<0.01$ \\
\hline $\mathrm{BMI}$ in $\mathrm{kg} / \mathrm{m}^{2}$, mean $(\mathrm{SD})$ & $20.4(5.8)$ & $32.9(12.2)^{\mathrm{a}}$ & $<0.01$ \\
\hline Asthma, $n(\%)$ & $3(27 \%)$ & $3(19 \%)$ & 0.66 \\
\hline Malignancy, $n(\%)$ & $0(0 \%)$ & $2(13 \%)$ & 0.50 \\
\hline Cardiac disease, $n(\%)$ & $1(9 \%)$ & $1(6 \%)$ & $>0.99$ \\
\hline Neurological disease, $n(\%)$ & $0(0 \%)$ & $2(13 \%)$ & 0.50 \\
\hline \multicolumn{4}{|l|}{ Outcomes } \\
\hline Length of stay in days, mean (SD) & $12.8(6.1)$ & $18.8(32.6)$ & 0.55 \\
\hline
\end{tabular}

Bold values indicate statistical significance $B M I$ body mass index, $S D$ standard deviation ${ }^{\mathrm{a}} n=14$; BMI was not available in two cases 
91\% (10/11) received intravenous furosemide. All children with MIS-C survived; one child with COVID-19 died of complications from acute myelogenous leukemia.

\section{Chest radiographs in children with COVID-19 versus those with MIS-C}

Analysis and categorization of the most severe chest radiograph from each patient with MIS-C versus those with COVID-19 are shown in Table 2. Selected chest radiographs from patients with COVID-19 and MIS-C are shown in Figs. 1 and 2, respectively. The percentage of normal baseline chest radiographs was greater in children with MIS-C (64\% [7/11]) than in children with COVID-19 (38\% [6/16]), but this did not reach significance ( $P$ value $=0.25$ ). Children with MIS-C did have a significantly higher number of chest radiographs obtained (6.3 [SD 5.1] vs. 2.8 [SD 2.6], $P$-value $=0.03$ ).
Children with MIS-C were significantly more likely to have an atypical categorization than children with COVID-19 (82\% [9/11] vs. $6 \%[1 / 16], P$-value $<0.01)$. The majority of children with MIS-C (73\% [8/11]) had both consolidation and ground glass opacities. Compared to children with COVID-19, children with MIS-C were significantly more likely to have lower zone predominance of pulmonary opacities $(100 \%$ [10/10] vs. $38 \%$ [5/13], $P$-value $<0.01)$ and pleural effusions $(82 \%$ [9/11] vs. $0 \%$ [0/16], $P$-value $<0.01)$. In children with MIS-C and pleural effusions, $67 \%$ (6/9) of the pleural effusions were bilateral.

\section{Additional imaging findings in MIS-C}

Additional imaging studies performed in patients with MIS-C are shown in Online Supplementary Material 1. In addition to chest radiographs, all children with MIS-C had echocardiograms performed, 64\% (7/11) had abdominal radiographs and
Table 2 Chest radiograph findings in pediatric patients with multisystem inflammatory syndrome in children (MIS-C) and coronavirus disease 2019 (COVID-19)

\begin{tabular}{|c|c|c|c|}
\hline & MIS-C $(n=11)$ & COVID-19 $(n=16)$ & $P$-value \\
\hline Baseline chest radiograph normal, $n(\%)$ & $7(64 \%)$ & $6(38 \%)$ & 0.25 \\
\hline Number of chest radiographs, mean (SD) & $6.3(5.1)$ & $2.8(2.6)$ & 0.03 \\
\hline Day of most severe radiograph, mean (SD) & $4.5(2.3)$ & $3.3(3.6)$ & 0.34 \\
\hline Severity score, mean (SD) & $3.6(1.4)$ & $2.9(2.4)$ & 0.39 \\
\hline \multicolumn{4}{|l|}{ COVID-19 classification } \\
\hline Typical, $n(\%)$ & $2(18 \%)$ & $4(25 \%)$ & $>0.99$ \\
\hline Indeterminate, $n(\%)$ & $0(0 \%)$ & $8(50 \%)$ & $<0.01$ \\
\hline Atypical, $n(\%)$ & $9(82 \%)$ & $1(6 \%)$ & $<0.01$ \\
\hline Negative, $n(\%)$ & $0(0 \%)$ & $3(19 \%)$ & 0.25 \\
\hline \multicolumn{4}{|l|}{ Opacity, $n(\%)$} \\
\hline Consolidation only, $n(\%)$ & $0(0 \%)$ & $0(0 \%)$ & $>0.99$ \\
\hline Ground glass opacities only, $n(\%)$ & $2(18 \%)$ & $8(50 \%)$ & 0.12 \\
\hline Both, $n(\%)$ & $8(73 \%)$ & $5(31 \%)$ & 0.05 \\
\hline None, $n(\%)$ & $1(9 \%)$ & $3(19 \%)$ & 0.62 \\
\hline \multicolumn{4}{|l|}{ Laterality of opacities ${ }^{\mathrm{a}}$} \\
\hline Right only, $n(\%)$ & $0(0 \%)$ & $0(0 \%)$ & $>0.99$ \\
\hline Left only, $n(\%)$ & $0(0 \%)$ & $2(15 \%)$ & 0.49 \\
\hline Bilateral, $n(\%)$ & $10(100 \%)$ & $11(85 \%)$ & 0.49 \\
\hline \multicolumn{4}{|l|}{ Distribution of opacities ${ }^{\mathrm{a}}$} \\
\hline Peripheral, $n(\%)$ & $4(40 \%)$ & $5(38 \%)$ & $>0.99$ \\
\hline Central, $n(\%)$ & $0(0 \%)$ & $2(15 \%)$ & 0.50 \\
\hline Neither, $n(\%)$ & $6(60 \%)$ & $6(46 \%)$ & 0.70 \\
\hline \multicolumn{4}{|l|}{ Predominance of opacities ${ }^{\mathrm{a}}$} \\
\hline Upper zone, $n(\%)$ & $0(0 \%)$ & $0(0 \%)$ & $>0.99$ \\
\hline Lower zone, $n(\%)$ & $10(100 \%)$ & $5(38 \%)$ & $<0.01$ \\
\hline Neither, $n(\%)$ & $0(0 \%)$ & $8(62 \%)$ & $<0.01$ \\
\hline Pleural effusion, $n(\%)$ & $9(82 \%)$ & $0(0 \%)$ & $<0.01$ \\
\hline
\end{tabular}

Findings are based on the most severe radiograph. Bold values indicate statistical significance. $S D$ standard deviation

${ }^{\text {a }} n=13$ for patients with COVID-19 and $n=10$ for patients with MIS-C; three patients with COVID-19 and one patient with MIS-C had no opacities by chest radiography 


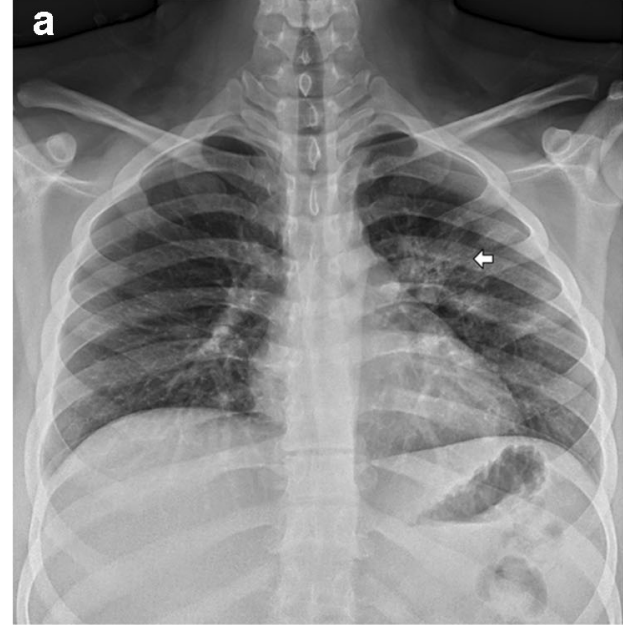

Fig. 1 Chest radiographs of children with coronavirus disease 2019 (COVID-19). a Posteroanterior chest radiograph in a 15-year-old boy with COVID-19 demonstrates a unilateral ground glass opacity (arrow) in the left lung. Categorization: indeterminate (based on a unilateral opacity). Severity score: 1 . b Anteroposterior chest radiograph in a 17-

$73 \%(8 / 11)$ had sonography performed, either of the abdomen $(55 \%[6 / 11])$, neck $(9 \%[1 / 11])$ or extremities $(27 \%$ [3/11]). Six children (55\%) had CT performed, either of the abdomen and pelvis $(36 \%[4 / 11])$, head $(18 \%[2 / 11])$, or face $(9 \%$ [1/11]). No children with MIS-C at our center had CT of the chest performed. Only one child had magnetic resonance imaging (MRI), and this was of the brain.

Imaging findings among children with MIS-C are summarized in Table 3 with imaging examples given in Fig. 3. Echocardiographic findings included depressed myocardial function $(64 \%$ [7/11]), valvular regurgitation $(64 \%$ [7/11]), and pericardial $(82 \%$ [9/11]) and pleural (82\% [9/11]) effusions. In addition to the pulmonary and cardiac findings, the majority of children who had abdominal imaging performed $(n=4)$ also had abnormalities including mesenteric adenopathy (75\% [3/4]), mesenteric edema (50\% [2/4]), bowel wall thickening (25\% [1/4]), ascites (57\% [4/7]) and gallbladder wall thickening (29\% [2/7]). Additionally, one child had cervical lymphadenopathy identified by neck ultrasound, and one child had a cytotoxic lesion of the corpus callosum on MRI.

\section{Discussion}

In this retrospective case series, radiographic features of MIS$\mathrm{C}$ on the most severe chest radiographs included pleural effusions $(82 \%$ [9/11]), pulmonary consolidations $(73 \%$ [8/11]) and ground glass opacities $(91 \%$ [10/11]). Children with MIS-C were significantly more likely than children with COVID-19 to develop pleural effusions ( $82 \%$ [9/11] vs. $0 \%$ [0/16], $P$-value $<0.01)$ and a lower zone predominance of pulmonary opacifications $(100 \%$ [10/10] vs. $38 \%$ [5/13], $P$ value $<0.01$ ). As all children with MIS-C in our study had

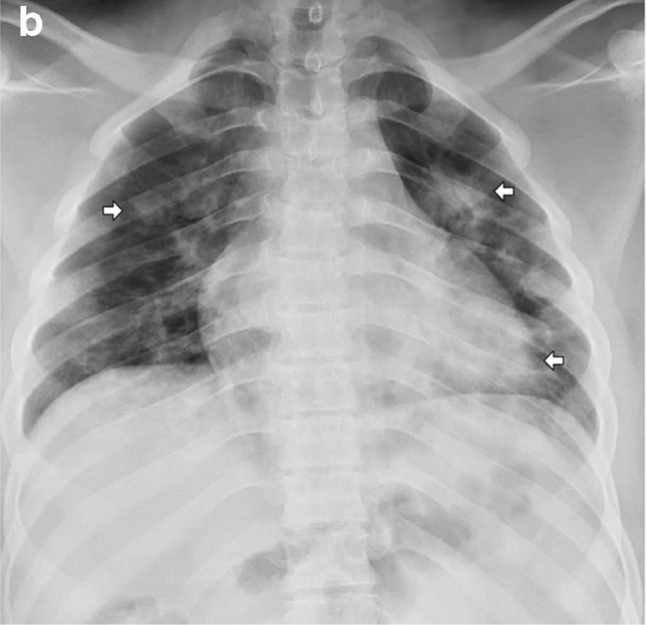

year-old boy with COVID-19 demonstrates bilateral multifocal consolidative and ground glass opacities (arrows). Categorization: indeterminate (based on multifocal opacities without a specific distribution). Severity score: 5

fluid-refractory shock requiring volume resuscitation and vasoactive medications, we suspect the evolution of pleural effusions and ground glass opacities during their hospitalizations were, in part, attributable to third spacing and pulmonary edema. This is consistent with observations previously described by Hameed et al. [12], who identified pleural effusions (11\% [4/35]) and pulmonary opacifications presumed to be edema $(31 \%$ [11/35]) among their cohort of children with MIS-C, although less frequently.

The majority of children with MIS-C also had depressed myocardial function by echocardiography (64\% [7/11]). Cardiac injury and myocarditis are known complications of MIS-C, and they can result in acute heart failure with cardiac decompensation in the setting of severe systemic inflammation and cytokine storm. In a case series of 35 children with MIS-C and acute heart failure, Belhadjer et al. [10] found that $80 \%$ required inotropic support and $28 \%$ required extracorporeal membrane oxygenation (ECMO). Although none of the patients with MIS-C in our cohort required ECMO, all required inotropic support, and the majority had evidence of myocardial dysfunction $(64 \%$ [7/11]), which likely contributed to their imaging findings. The extent to which volume overload, depressed myocardial function, and an overwhelming systemic inflammatory response contributed to pleural effusions and third spacing in our study is unclear, as all processes appeared to occur simultaneously. This leads us to suspect that the pathogenesis of the disease process leading to these findings is multifactorial.

We categorized chest radiographs as typical, indeterminate, atypical or negative for findings of COVID-19, according to the classification initially described for use with $\mathrm{CT}$ in adults and subsequently adapted for use in pediatric chest radiography by Foust et al. [13, 14]. We found that children with MIS$\mathrm{C}$ were significantly more likely to have an atypical 

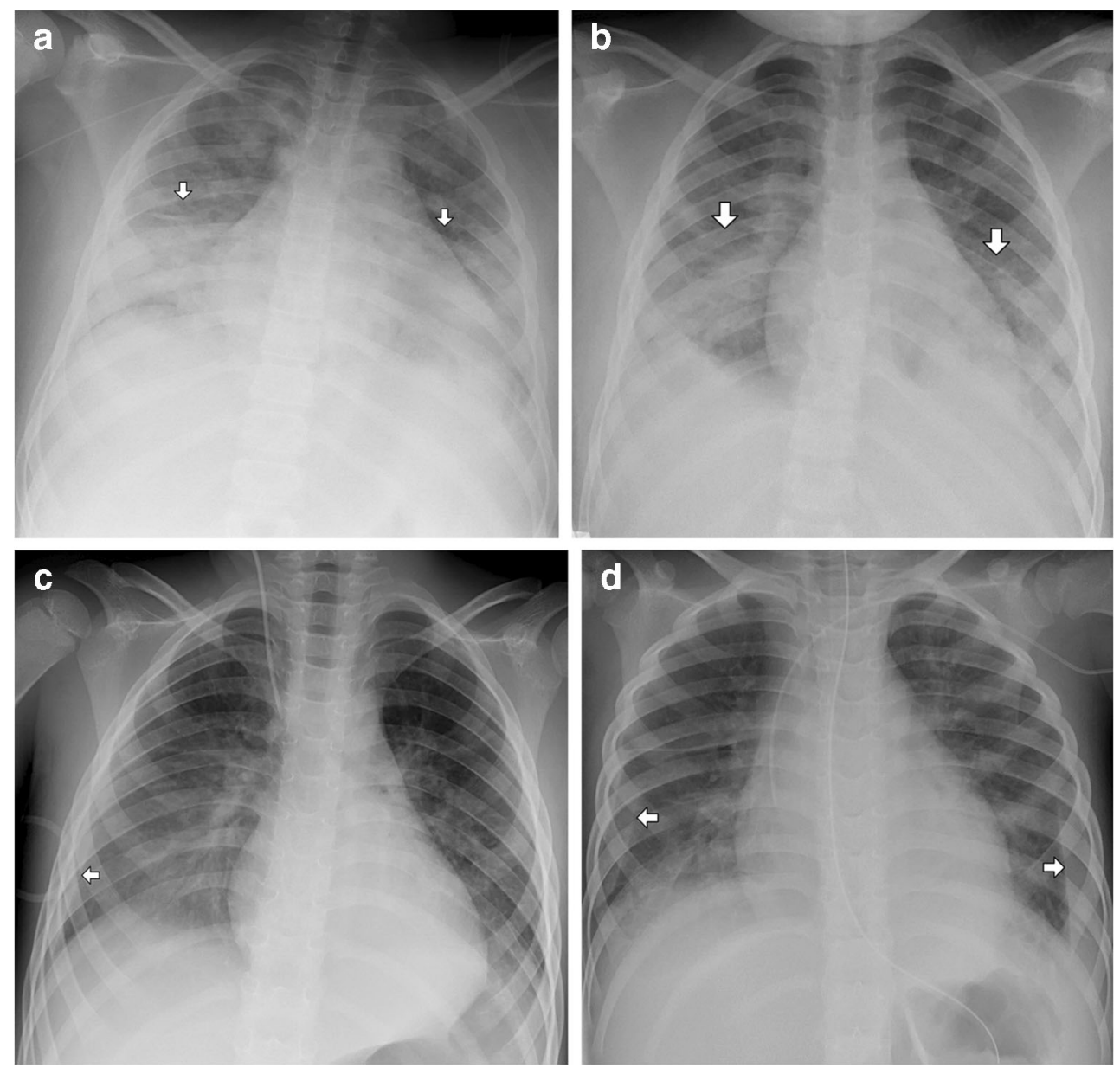

Fig. 2 Chest radiographs of children with multisystem inflammatory syndrome in children (MIS-C). a Anteroposterior (AP) chest radiograph in a 12-year-old boy with MIS-C demonstrates bilateral consolidative and ground glass opacities (arrows) in a peripheral lower zone distribution. Categorization: typical (based on bilateral peripheral opacities). Severity score: 4. b AP chest radiograph in a 10-year-old girl demonstrates bilateral consolidative and ground glass opacities (arrows) in a peripheral lower zone distribution. Categorization: typical. Severity

categorization than children with COVID-19, primarily because of pleural effusions. Therefore, $82 \%(9 / 11)$ of radiographs in patients with MIS-C were atypical, compared to only $6 \%(1 / 16)$ of radiographs in patients with COVID-19 $(P$-value $<0.01)$. However, two of the children with MIS-C had chest radiographs that were typical for COVID-19, indicating that MIS-C and COVID-19 radiographic findings can overlap in some cases.

We additionally compared the type and distribution of chest radiograph airspace opacities found among children with MIS-C versus those with COVID-19 and calculated a severity score using a simplified version of the Radiographic Assessment of Lung Edema Score, which has previously been used by Wong et al. [15] to describe chest radiograph findings in adults with COVID-19 [16]. All (10/10) of the pulmonary opacities found in children with MIS-C were lower zone

score: 4. c AP chest radiograph in a 4-year-old boy demonstrates a right-side pleural effusion (arrow), a right-side ground glass opacity and a left-side consolidative opacity. The opacities are in a lower zone distribution. Categorization: atypical (based on the presence of a pleural effusion). Severity score: 4 . d AP chest radiograph in a 3-year-old boy demonstrates bilateral pleural effusions (arrows) and bilateral ground glass opacities in a peripheral lower zone distribution. Categorization: atypical (based on the presence of a pleural effusion). Severity score: 4

predominant, compared to $38 \%(5 / 13)$ of the pulmonary opacities in children with COVID-19. For context, when Wong et al. [15] applied this analysis to adults with COVID-19, they found that $50 \%(32 / 64)$ of patients had lower zone distribution, 50\% (32/64) had bilateral involvement and 3\% (2/64) had pleural effusions. The mean chest radiograph severity score in our study was 3.6 (SD 1.4) for children with MIS-C compared to 2.9 (SD 2.4) for children with COVID-19. These differences were not statistically different $(P$-value $=0.39)$, and according to Wong et al. [15], scores tend to vary widely during the course of hospitalization.

Abdominal imaging in patients with MIS-C demonstrated inflammation including mesenteric adenopathy (75\% [3/4]), mesenteric edema $(50 \%[2 / 4])$, ascites $(57 \%$ [4/7]), bowel wall thickening (25\% [1/4]) and gallbladder wall thickening (29\% [2/7]). These imaging findings are consistent with the 
Table 3 Additional imaging findings in patients with multisystem inflammatory syndrome in children (MIS-C) during hospitalization

MIS-C

$\begin{array}{ll}\text { Echocardiogram ( } n=11) & 7(64 \%) \\ \text { Decreased myocardial function, } n(\%) & 7(64 \%) \\ \text { Valvular regurgitation (mild to moderate), } n(\%) & 9(82 \%) \\ \text { Pleural effusions, } n(\%) & 9(82 \%) \\ \text { Pericardial effusion, } n(\%) & 0(0 \%) \\ \text { Coronary artery dilation, } n(\%) & \\ \text { Abdomen (CT, } n=4) & 3(75 \%) \\ \text { Mesenteric adenopathy, } n(\%) & 2(50 \%) \\ \text { Mesenteric edema, } n(\%) & 1(25 \%) \\ \text { Bowel wall thickening, } n(\%) & 4(57 \%) \\ \text { Abdomen (CT or US, } n=7) & 2(29 \%) \\ \quad \begin{array}{l}\text { Ascites, } n(\%) \\ \text { Gallbladder wall thickening, } n(\%)\end{array} \\ \text { Central nervous system (MRI, } n=1) & 1(100 \%) \\ \quad \text { Cytotoxic lesion of the corpus callosum, } n(\%) & 1(100 \%) \\ \text { Other (US, } n=1) & \\ \text { Cervical adenopathy, } n(\%) & \end{array}$

clinical features of MIS-C, as 100\% (11/11) of children in our cohort had gastrointestinal symptoms at the time of presentation and these were distinguishing features of MIS-C compared to COVID-19 ( $P$-value $=0.05)$. Other recent studies have also found a predominance of gastrointestinal symptoms in MIS-C [5, 6], and Hameed et al. [12] similarly observed signs of gastrointestinal inflammation in a proportion of children with MIS-C who had abdominal imaging performed. One patient who had a palpable neck mass was found to have cervical lymphadenopathy on neck ultrasound, which has been reported in up to $10 \%$ of children with MIS-C. Another patient, who had signal abnormality in the corpus callosum, was recently described [17].

Limitations of our study include its small sample size and retrospective nature. Although our study found that pleural effusions are much more common in patients with MIS-C than in patients with COVID-19, pleural effusions can be seen with COVID-19. Also, our study focused on the most severe chest radiograph. We did this to maximize the sensitivity of the chest imaging findings on radiography, but this also limits our study's ability to evaluate the evolution of imaging findings over time. Additionally, not all patients had abdominal imaging studies, and thus the abdominal imaging findings could not be completely characterized. Further studies are needed to more fully characterize imaging findings of MIS$\mathrm{C}$ and to compare them to other inflammatory syndromes, including Kawasaki disease and toxic shock syndrome. Although the imaging findings identified in patients with MIS-C in this study could not be considered pathognomonic,
Fig. 3 Additional imaging findings in children with multisystem inflammatory syndrome in children (MIS-C). a Axial CT of the abdomen and pelvis (volume computed tomography dose index $\left[\mathrm{CTDI}_{\mathrm{vol}}\right]$ $16.23 \mathrm{mGy}$ ) in a 12 -year-old boy with MIS-C demonstrates an enlarged mesenteric lymph node $(x)$, mesenteric edema (star) and bowel wall thickening of the colon (arrow). b Transverse ultrasound of the abdomen in a 5year-old girl with MIS-C demonstrates an enlarged mesenteric lymph node measuring $3.4 \mathrm{~cm}$ (denoted by calipers). c Transverse ultrasound of the gallbladder in an 8-year-old boy demonstrates gallbladder wall thickening (arrow)
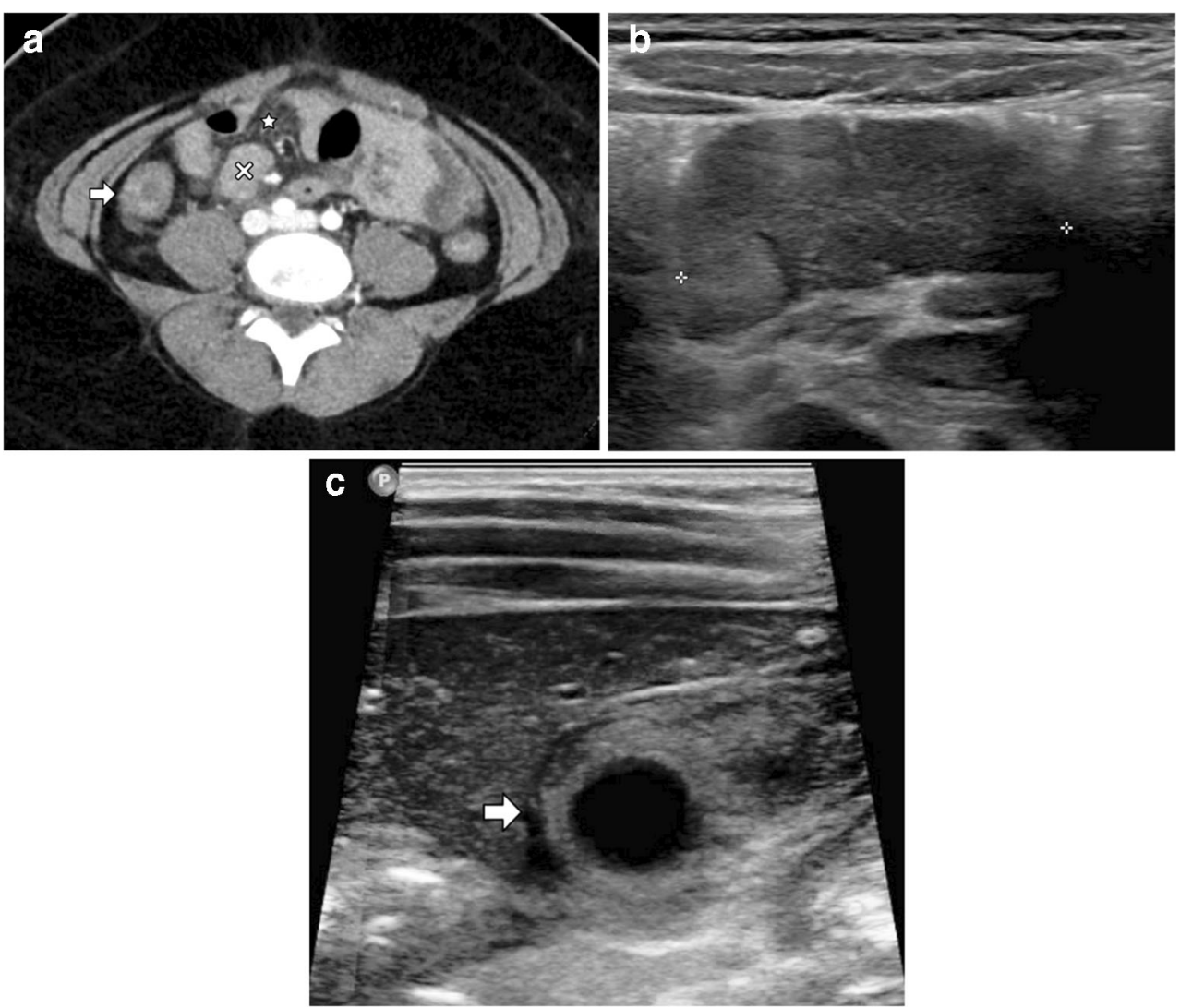
much remains to be learned about this novel clinical syndrome. The current CDC case definition of MIS-C is quite broad, and could likely be refined as we learn more about the characteristic clinical, laboratory and radiographic features of MIS-C. Radiologists should be aware of any differentiating imaging findings that may alert clinicians to this diagnosis, as its management is unique from other systemic inflammatory conditions and its outcomes can be severe.

\section{Conclusion}

Distinguishing radiographic features of MIS-C include pleural effusions, bilateral pulmonary consolidations with lower zone predominance and intra-abdominal inflammatory changes. Identifying these findings in a pediatric patient in the era of COVID-19 should prompt the radiologist to consider MIS-C in the differential diagnosis. Elucidating the distinguishing radiographic features of MIS-C may help refine the case definition and expedite diagnosis and treatment.

Supplementary Information The online version contains supplementary material available at https://doi.org/10.1007/s00247-020-04921-9.

Acknowledgments Christina A. Rostad receives funding from the Center for Childhood Infections and Vaccines and from the Children's Pediatric Trust.

\section{Compliance with ethical standards}

Conflicts of interest Christina A. Rostad has received royalties unrelated to this manuscript to Emory University from Meissa Vaccines Inc. She has also received funds to her institution to conduct clinical research unrelated to this manuscript from MedImmune, Regeneron, Paxvax, Pfizer, GSK, Merck, Novavax, Sanofi-Pasteur and Micron.

\section{References}

1. World Health Organization (2020) Coronavirus disease 2019 (COVID-19) Situation Report - 51. https://www.who.int/docs/ default-source/coronaviruse/situation-reports/20200311-sitrep-51covid-19.pdf?sfvrsn=1ba62e57_10. Accessed 4 July 2020

2. Ludvigsson JF (2020) Systematic review of COVID-19 in children shows milder cases and a better prognosis than adults. Acta Paediatr 109:1088-1095

3. Riphagen S, Gomez X, Gonzalez-Martinez C et al (2020) Hyperinflammatory shock in children during COVID-19 pandemic. Lancet 395:1607-1608
4. Verdoni L, Mazza A, Gervasoni A et al (2020) An outbreak of severe Kawasaki-like disease at the Italian epicentre of the SARSCoV-2 epidemic: an observational cohort study. Lancet 395:17711778

5. Feldstein LR, Rose EB, Horwitz SM et al (2020) Multisystem inflammatory yndrome in U.S. children and adolescents. N Engl J Med 383:334-346

6. Dufort EM, Koumans EH, Chow EJ et al (2020) Multisystem inflammatory syndrome in children in New York state. N Engl J Med 383:347-358

7. Centers for Disease Control and Prevention (2020) Multisystem inflammatory syndrome in children (MIS-C) associated with coronavirus disease 2019 (COVID-19). https://emergency.cdc.gov/han/ 2020/han00432.asp. Accessed 4 July 2020

8. Viner RM, Whittaker E (2020) Kawasaki-like disease: emerging complication during the COVID-19 pandemic. Lancet 395:17411743

9. Whittaker E, Bamford A, Kenny J et al (2020) Clinical characteristics of 58 children with a pediatric inflammatory multisystem syndrome temporally associated with SARS-CoV-2. JAMA 324: 259-269

10. Belhadjer Z, Méot M, Bajolle F et al (2020) Acute heart failure in multisystem inflammatory syndrome in children (MIS-C) in the context of global SARS-CoV-2 pandemic. Circulation. https://doi. org/10.1161/CIRCULATIONAHA. 120.048360

11. Shelmerdine SC, Lovrenski J, Caro-Domínguez P et al (2020) Coronavirus disease 2019 (COVID-19) in children: a systematic review of imaging findings. Pediatr Radiol 50:1217-1230

12. Hameed S, Elbaaly H, Reid CEL et al (2020) Spectrum of imaging findings on chest radiographs, US, CT, and MRI images in multisystem inflammatory syndrome in children (MIS-C) associated with COVID-19. Radiology 202543

13. Simpson S, Kay FU, Abbara S et al (2020) Radiological Society of North America expert consensus statement on reporting chest CT findings related to COVID-19. Endorsed by the Society of Thoracic Radiology, the American College of Radiology, and RSNA secondary publication. J Thorac Imaging 35:219-227

14. Foust AM, Phillips GS, Chu WC et al (2020) International expert consensus statement on chest imaging in pediatric COVID-19 patient management: imaging findings, imaging study reporting and imaging study recommendations. Radiology: Cardiothoracic Imaging 2:e200214

15. Wong HYF, Lam HYS, Fong AH-T et al (2020) Frequency and distribution of chest radiographic findings in patients positive for COVID-19. Radiology 296:E72-E78

16. Warren MA, Zhao Z, Koyama T et al (2018) Severity scoring of lung oedema on the chest radiograph is associated with clinical outcomes in ARDS. Thorax 73:840-846

17. Lin J, Lawson EC, Verma S et al (2020) Cytotoxic lesion of the corpus callosum in an adolescent with multisystem inflammatory syndrome and SARS-CoV-2 infection. AJNR Am J Neuroradiol. https://doi.org/10.3174/ajnr.A6755

Publisher's note Springer Nature remains neutral with regard to jurisdictional claims in published maps and institutional affiliations. 